\title{
Family values: \\ Maternal care in rattlesnakes is more than mere attendance
}

\author{
Melissa Amarello', Jeffrey J. Smith, \& John Slone \\ SocialSnakes.blogspot.com \\ ${ }^{1}$ School of Life Sciences, Arizona State University
}
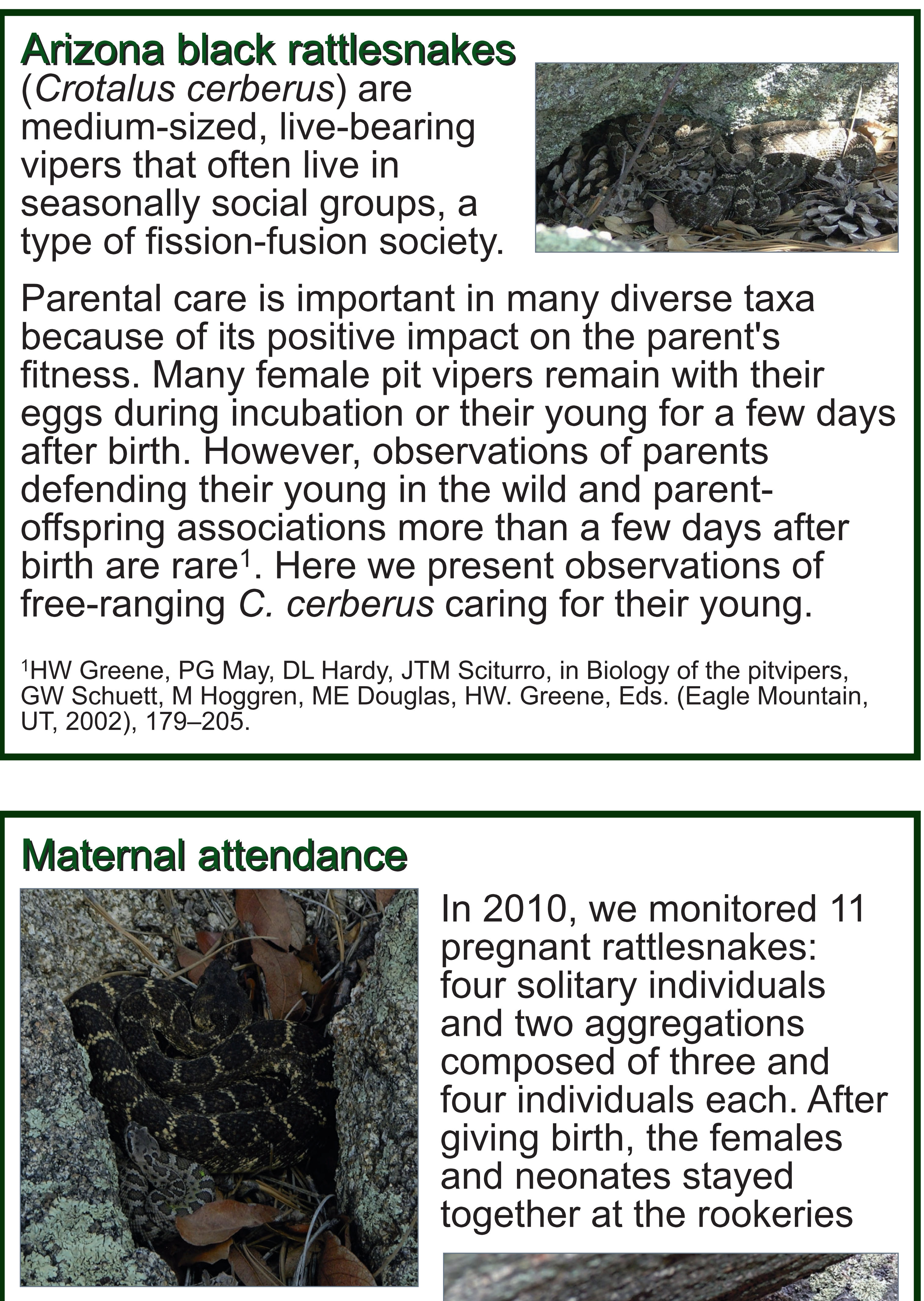

for up to 12 days. While the presence of an adult rattlesnake may deter some predators, we observed defensive behavior after, but not before, birth.

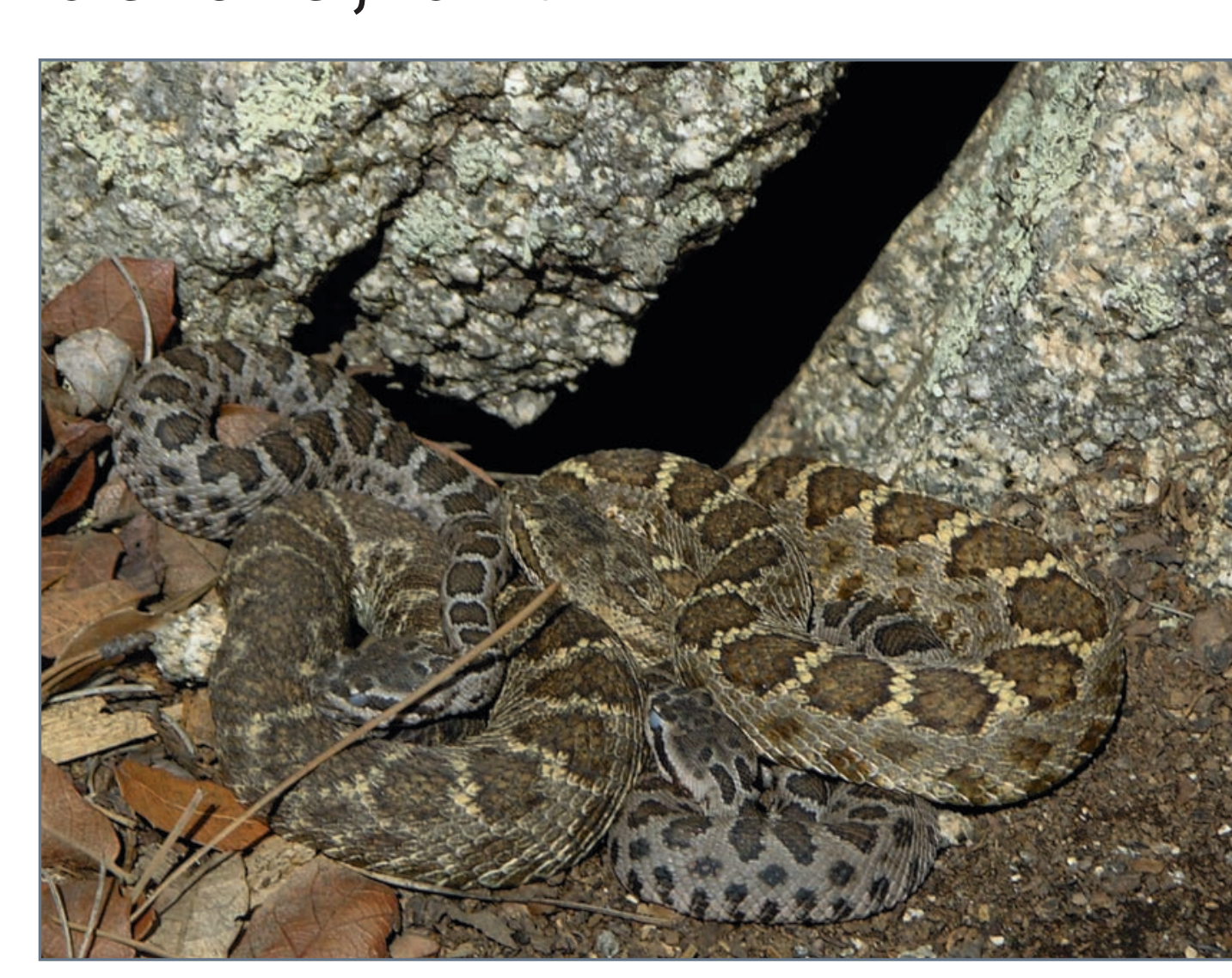

n 2010, we monitored 11 pregnant rattlesnakes: four solitary individuals and two aggregations composed of three and four individuals each. After giving birth, the females and neonates stayed together at the rookeries

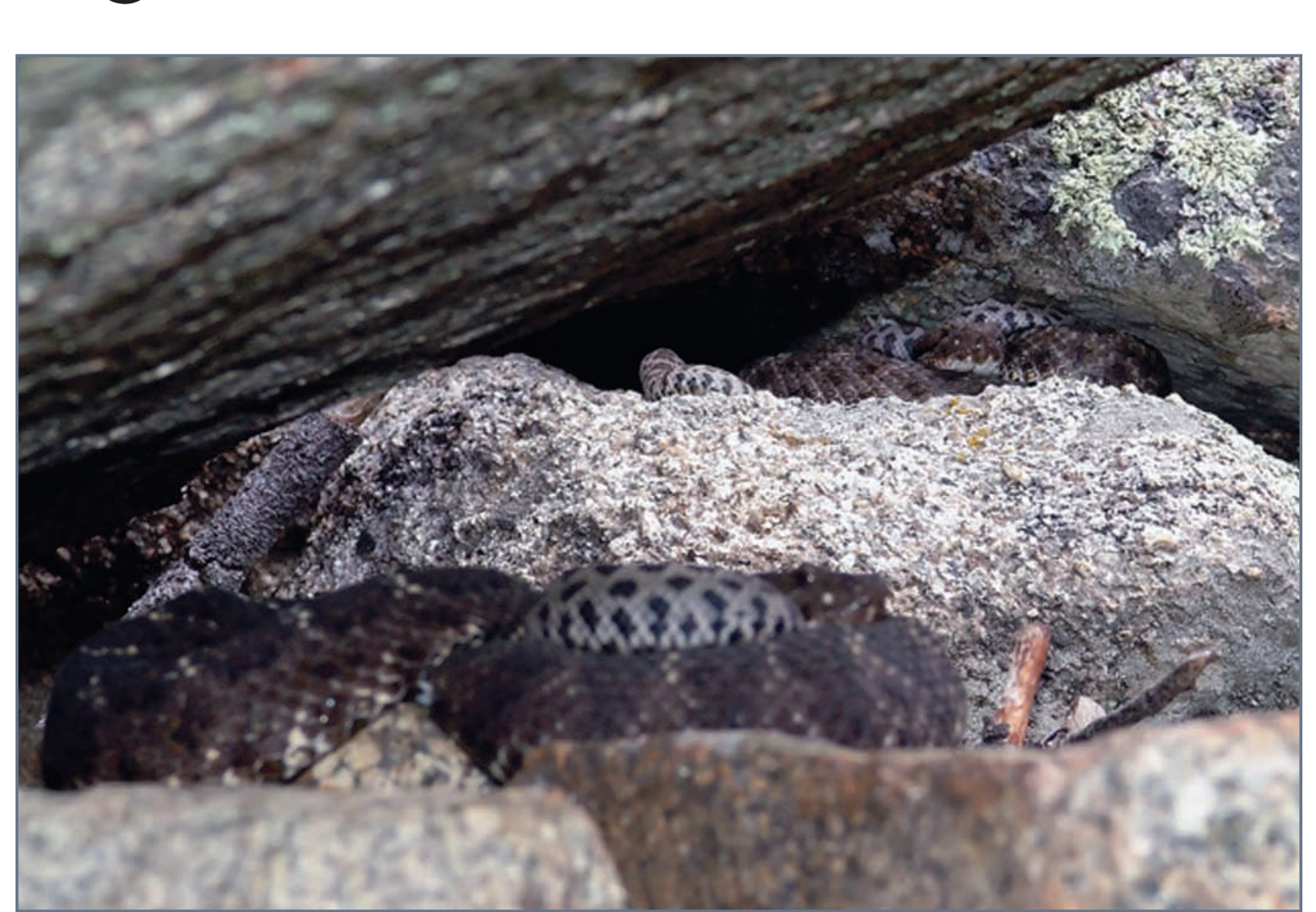

Snake E basking with neonates (left) quickly takes on a defensive posture at the approach of a human predator (right).

\section{A rattlesnake helper?}

On 30 August 2010 we observed what appeared to be an adult rattlesnake (Snake $P$ ) discouraging a neonate (Snake $\mathrm{N}$ ) from potential exposure to a human predator. Snake $\mathrm{P}$ was pregnant at this time, so Snake $\mathrm{N}$ had a different mother. Perhaps this is why some female rattlesnakes aggregate during gestation and after the birth of their offspring.

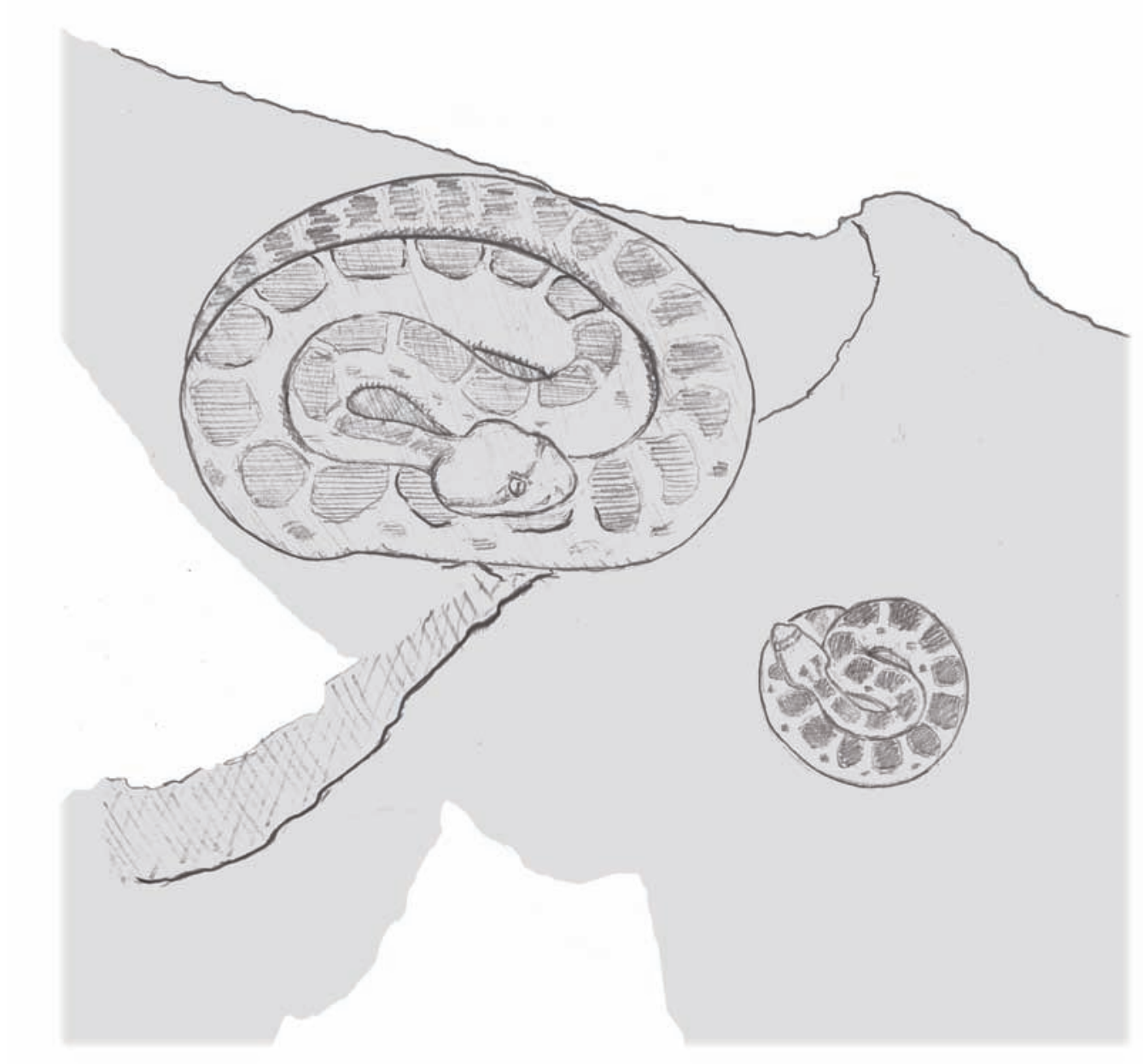

15:27 Snake $P$ (adult female) and Snake N (neonate) are at rest in a shaded rock shelter.

15:28 Snake $\mathrm{N}$ moves restlessly in cover and then begins to move toward open ground.

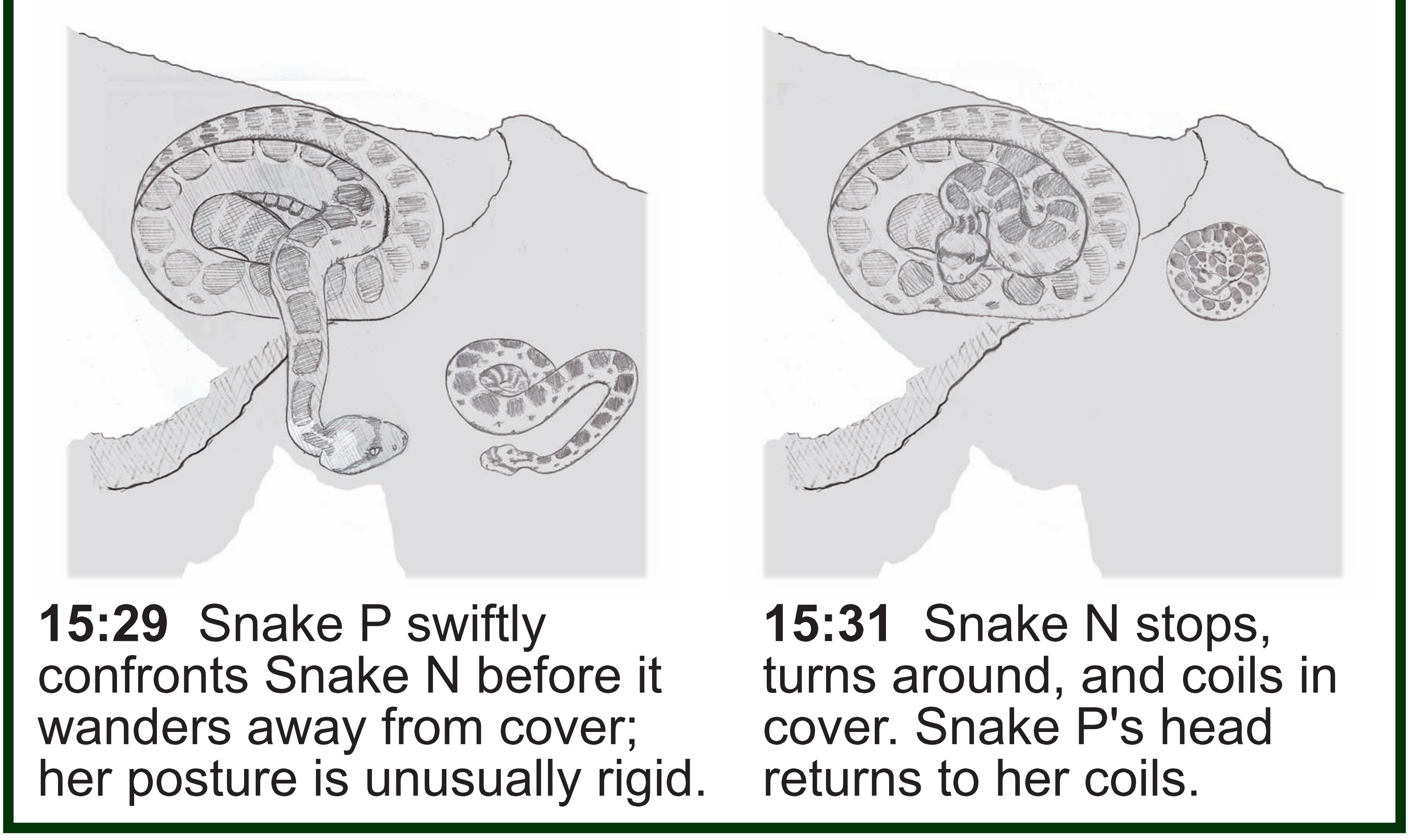

Vigilance from cover Often neonates were on the surface without an adult visible. On five occasions when we approached too closely, the nearest adult either rattled from within her

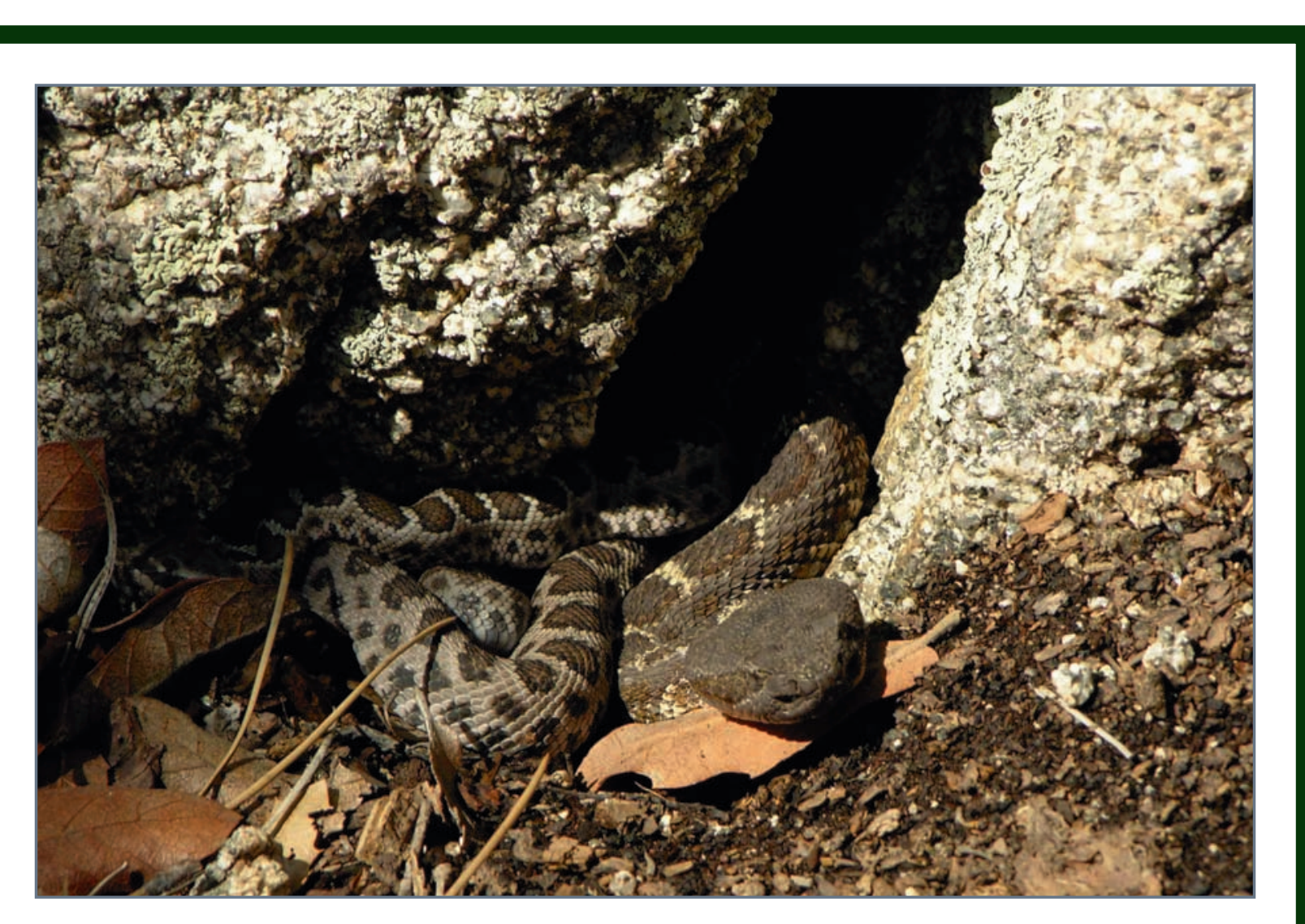

hiding place or came out to investigate the threat. Clouded eyes and naivete make neonates particularly vulnerable, so they often retreat into nearby shelter when alerted by an adult rattlesnake.

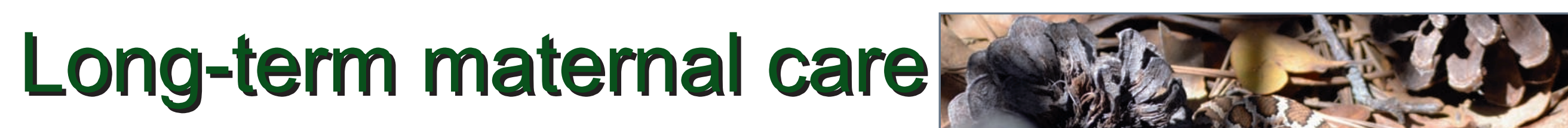
We identified individual rattlesnakes using pattern aberrancies to minimize capturing them and disturbing their behavior. Snake W $\&$ her 2010
offspring in April 2011

Like other rattlesnakes, neonate $C$. cerberus use the same winter hibernaculum (den) as their mother. During the spring emergence period we observed females basking near their neonates on four occasions and following their neonates as they explored the area around the den on one occasion.

\section{Potential role of males Adult males visited two rot.

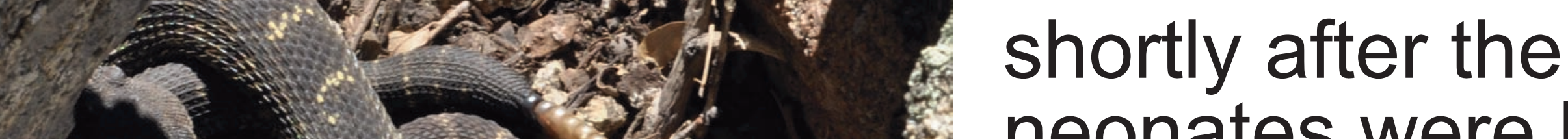 1 - 12 , neonates were born. In

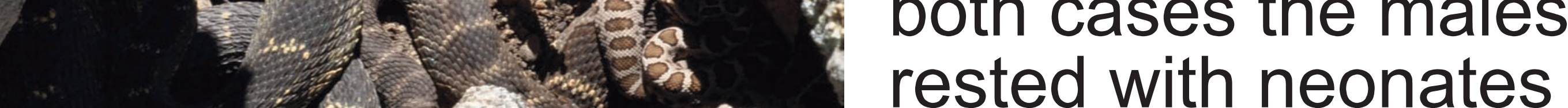 3. Adut male (eft) with and allowed them to

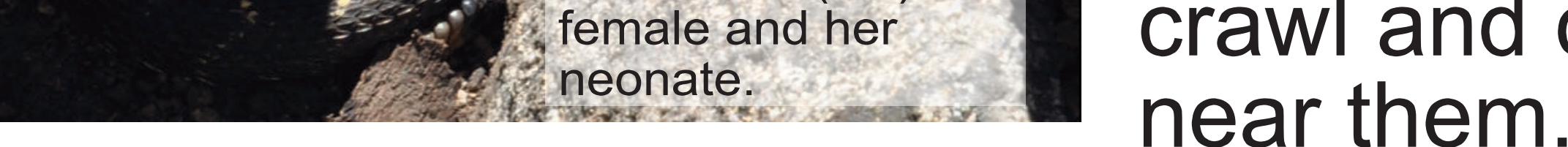

During these interactions, we did not observe any defensive behavior by the males or reproductive behavior between the males and females.

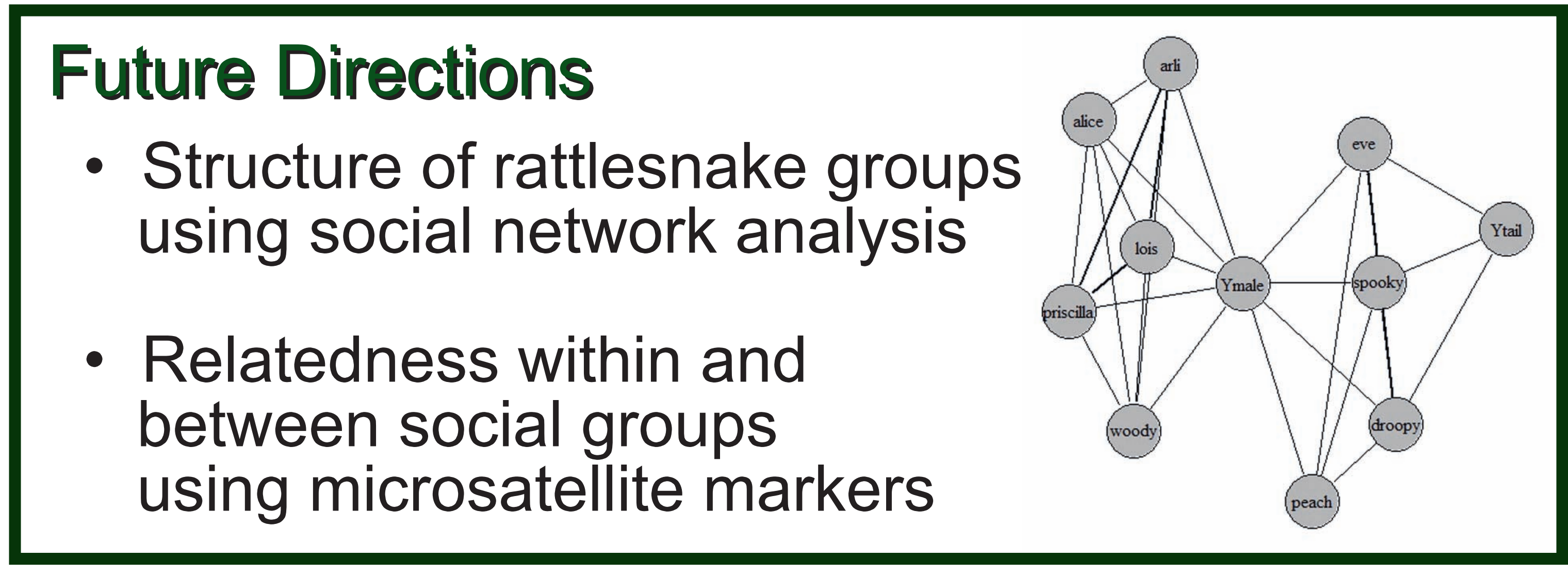

\section{Acknowledgements}

. McGraw, K. Moeller, K. Pegram, R. Repp, C. Smith, and $\mathrm{L}$. Taylor for field assistance and input on earlier versions of this poster. Permits were issued by the Arizona Game and Fish Department (SP796730 \& SP729143) and a protocol approved by the ASU Institutional Animal Care and Use Committees; funding was provided to MA by a National Science Foundation GRFP and the Chicago Herpetological Society. 